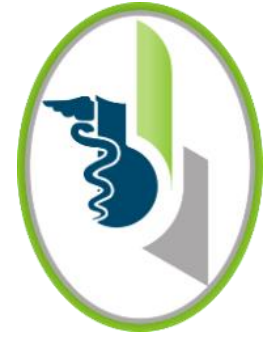

ACCESO $\odot$ ABIERTO

Para citaciones: Pérez, C., Murillo, M.,

Redondo, C., Redondo, K. (2019).

Endometritis xantogranulomatosa

asociada a carcinoma escamocelular de

cérvix: presentación de un caso y

revisión de la literatura. Revista

Ciencias Biomédicas, 8(2), 105-110.

Recibido: 5 de junio de 2019

Aprobado: 26 de junio de 2019

Autor de correspondencia:

Camilo Pérez Montiel

capm2908@gmail.com

Editor: Inés Benedetti. Universidad de Cartagena-Colombia.

Copyright: C 2019. Pérez, C., Murillo, M., Redondo, C., Redondo, K. Este es un artículo de acceso abierto, distribuido bajo los términos de la licencia https://creativecommons.org/licenses/bync-sa/4.0/ la cual permite el uso sin restricciones, distribución y reproducción en cualquier medio, siempre y cuando el original, el autor y la fuente sean acreditados.

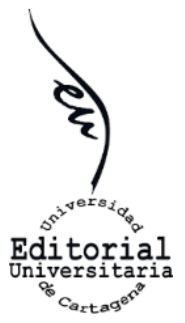

\section{Endometritis xantogranulomatosa asociada a carcinoma escamocelular de cérvix: presentación de un caso y revisión de la literatura}

\author{
Xanthogranulomatous endometritis associated with \\ squamous cell carcinoma of the cervix: case report and literature \\ review.
}
Pérez Montiel Camilo ${ }^{1}$, Murillo Salas María ${ }^{1}$, Redondo Bermúdez César ${ }^{1,2}$, Katherine Redondo de Oro ${ }^{1,2}$

\begin{abstract}
${ }^{1}$ Sección Patología. Facultad de Medicina. Universidad de Cartagena-Colombia
${ }^{2}$ Servicio de Anatomía Patológica, E.S.E. Hospital Universitario del Caribe, Cartagena, Colombia
\end{abstract}

\section{RESUMEN}

Introducción: la endometritis xantogranulomatosa es un proceso inflamatorio raro del endometrio, que se caracteriza por la presencia de abundantes histiocitos espumosos asociados a células inflamatorias mixtas. La importancia de este hallazgo histopatológico es su asociación con neoplasias malignas de origen endometrial y cervical, por lo cual el patólogo debe realizar un correcto abordaje morfológico e inmunohistoquímico. Se presenta un caso de endometritis xantogranulomatosa asociado a carcinoma escamocelular mal diferenciado de cérvix.

Caso Clínico: paciente femenina de 75 años de edad, con cuadro clínico de seis meses de evolución caracterizado por salida de líquido claro por genitales externos asociado a dolor pélvico intermitente. Ecográficamente se observa aumento de tamaño del útero, a expensas de acumulación de líquido con un volumen de $700 \mathrm{cc}$ y estenosis cervical. Se realiza biopsia endometrial guiada por histeroscopia con reporte histopatológico e inmunohistoquímico de endometritis xantogranulomatosa asociado a carcinoma escamocelular mal diferenciado de cérvix.

Conclusión: la endometritis xantogranulomatosa es un hallazgo histopatológico poco frecuente en el endometrio, lo cual es un reto diagnóstico para el patólogo debido a la asociación con neoplasias malignas de origen endometrial y cervical.

Palabras Clave: Endometritis xantogranulomatosa; carcinoma escamocelular; inflamación xantogranulomatosa.
ABSTRACT
Introduction: xanthogranulomatous endometritis is a rare inflammatory process of the endometrium characterized by the presence of abundant foamy histiocytes associated with mixed inflammatory cells. The importance of this histopathological finding is the association with malignant neoplasms of endometrial and cervical origin, for which the pathologist must carry out a correct morphological and immunohistochemical approach. A case of xanthogranulomatous endometritis 
associated with poorly differentiated squamous cell carcinoma of the cervix is presented.

Case Report: a 75-year-old female patient with a clinical picture of six months of evolution characterized by leakage of clear fluid from external genitalia associated with intermittent pelvic pain. Ultrasonographically, an increase in the uterus is observed at the expense of fluid accumulation with a volume of $700 \mathrm{cc}$ and cervical stenosis. Hysteroscopy-guided endometrial biopsy was performed with histopathological and immunohistochemical report of xanthogranulomatous endometritis associated with |poorly differentiated squamous cell carcinoma of the cervix.

Conclusion: xanthogranulomatous endometritis is a rare histopathological finding in the endometrium, which is a diagnostic challenge for the pathologist due to the association with malignant neoplasms of endometrial and cervical origin.

Key Words: Xanthogranulomatous endometritis; squamous cell carcinoma; xanthogranulomatous inflammation.

\section{INTRODUCCIÓN}

La respuesta inflamatoria xantogranulomatosa es un tipo de inflamación crónica poco frecuente, que se caracteriza por la presencia de abundantes histiocitos espumosos cargados de lípidos asociados con múltiples células inflamatorias (1). La inflamación xantogranulomatosa es más frecuente encontrarla en órganos como el riñón y la vesícula biliar (2). La endometritis xantogranulomatosa es un proceso inflamatorio raro e infrecuente del endometrio, con poco más de 20 casos reportados en la literatura inglesa. Clínicamente suele presentarse en mujeres postmenopáusicas que cursan con piometra o hematometra y se asocia a entidades como el carcinoma de endometrio, la hiperplasia endometrial y la estenosis cervical (3). El objetivo del siguiente manuscrito es presentar un caso de Endometritis Xantogranulomatosa asociada a carcinoma escamocelular de cérvix.

\section{REPORTE DE CASO}

Paciente femenina de 75 años de edad, con cuadro clínico de seis meses de evolución caracterizado por salida de líquido claro por los genitales y dolor pélvico intermitente. Antecedentes familiares: madre con cáncer de mama a los 45 años de edad y hermana con cáncer de estómago a los 50 años de edad. Como antecedentes personales, refiere hipertensión arterial y diabetes mellitus tipo 2, y en los antecedentes ginecoobstétricos refiere: G4P4A0, menarquia a los 14 años, ciclos regulares de 4 x 28, fecha de última menstruación a los 48 años edad, sin citología cervicouterina previa. Nunca planificó. Al examen físico, los hallazgos positivos fueron dolor a la palpación superficial y profunda del abdomen, útero aumentado de tamaño para 18 semanas de edad gestacional, doloroso y poco móvil. Al examen genitourinario, no se observó borramiento del fondo de saco vaginal, tampoco celes, ni lesiones evidentes en exocérvix. Se le realiza ecografía transvaginal que reporta: útero en anteroversión aumentado de tamaño a expensas de líquido denso de aproximadamente $700 \mathrm{cc}$, el cérvix midió 30 x 24 $\mathrm{mm}$, las paredes uterinas midieron $2-3 \mathrm{~mm}$ de grosor, sin lesiones intracavitarias. La histerometría reportó dimensiones de 189 x 82 x 72 mm, concluyendo gran hematometra secundario a estenosis del cérvix. La paciente posteriormente se lleva a biopsia endometrial guiada por histeroscopia hallándose orificio cervical externo e interno estenóticos. Se procede a tomar biopsia de secreción sanguinolenta en cavidad endometrial. Histopatológicamente se evidencia pérdida de la arquitectura del endometrio a expensas de un abundante infiltrado inflamatorio de histiocitos espumosos cargados de lípidos, junto con 
polimorfonucleares neutrófilos, linfocitos y plasmocitos, adyacente se observaron sábanas de células tumorales de linaje epitelial con núcleos hipercromáticos, pleomórficos y frecuentes figuras de mitosis (Figura 1). Se realiza el diagnóstico de Endometritis Xantogranulomatosa asociada a carcinoma mal diferenciado, por lo cual se llevan a cabo estudios de inmunohistoquímica, que reportan positividad para p63, CK 5/6 y p16 en las células tumorales (Figura 2) y negatividad para receptores de estrógeno y vimentina (Figura 3).

Concluyéndose, como diagnóstico definitivo un carcinoma escamocelular mal diferenciado del cérvix.
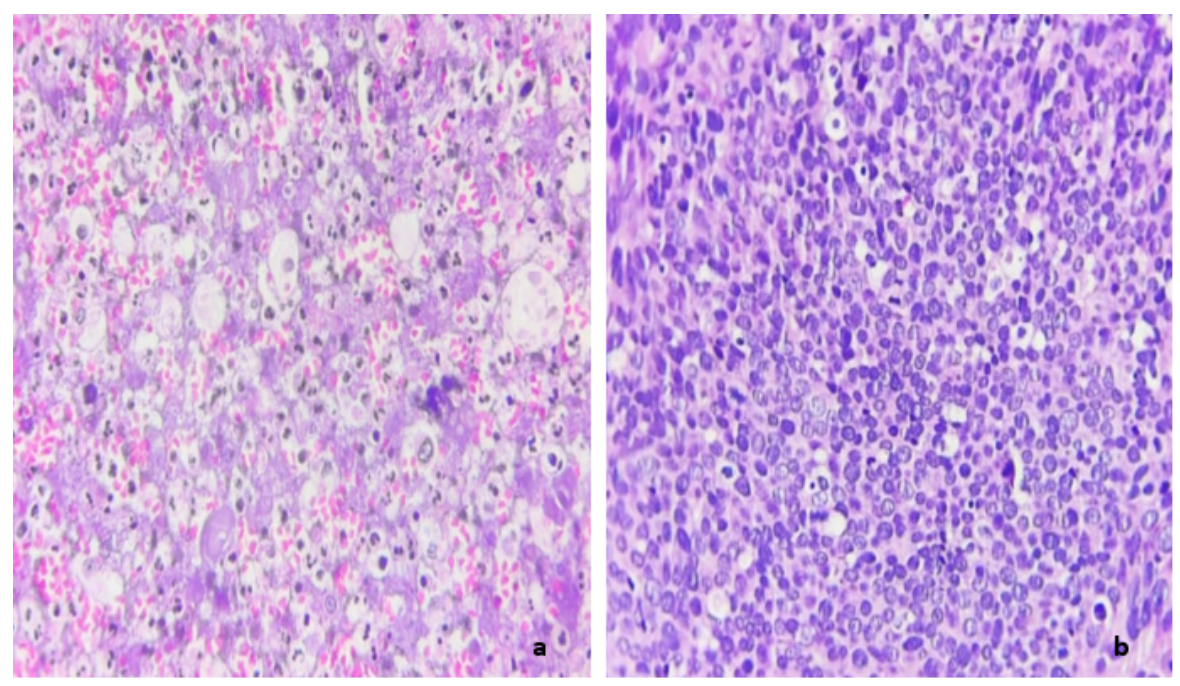

Figura 1. Endometritis Xantogranulomatosa asociada a sábanas de células tumorales epiteliales $\mathrm{Se}$ evidencia pérdida de la arquitectura normal del endometrio, abundantes histiocitos espumosos asociado a polimorfonucleares neutrófilos, linfocitos y plasmocitos (a). Células de linaje epitelial mal diferenciadas con núcleos pleomórficos y figuras de mitosis (b). Hematoxilina y Eosina 40X.
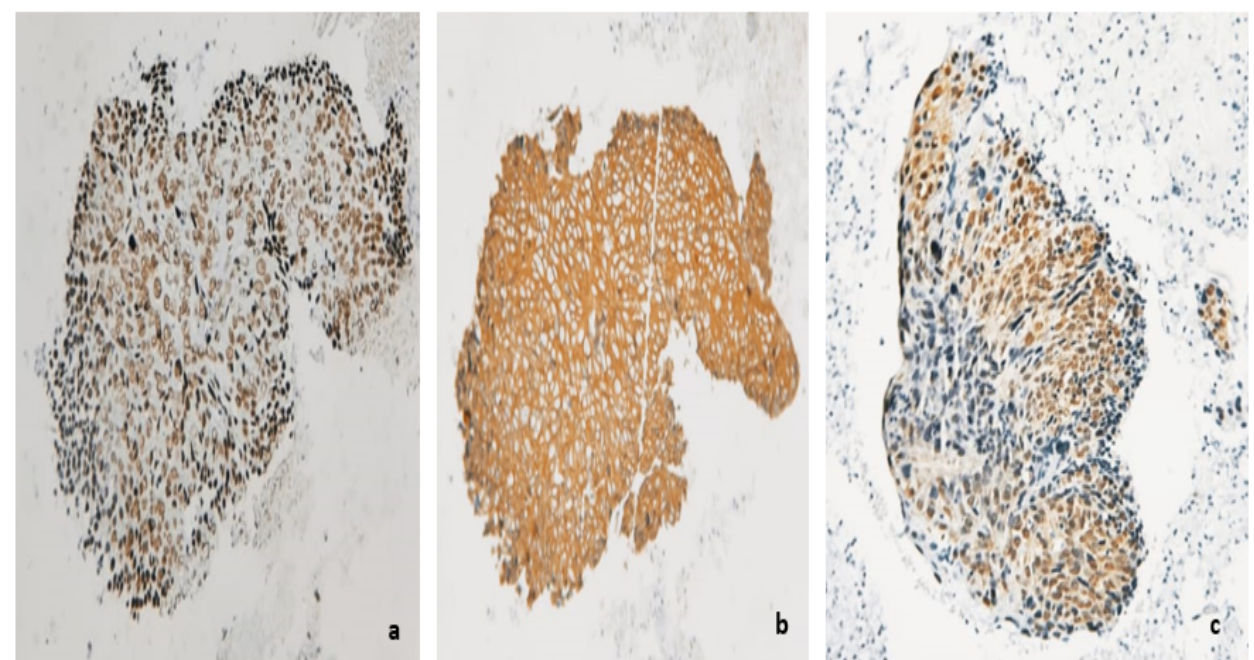

Figura 2. Inmunohistoquímica P63, CK5/6 y P16. Se observan cortes de tejido endometrial, con tinción nuclear fuerte para p63 en las células tumorales (a). Tinción citoplasmática y membranosa fuerte para CK 5/6 en las células tumorales (b). Tinción nuclear fuerte para p16 en las células tumorales (c). Inmunohistoquímica: p63, CK 5/6, p16, 40X. 

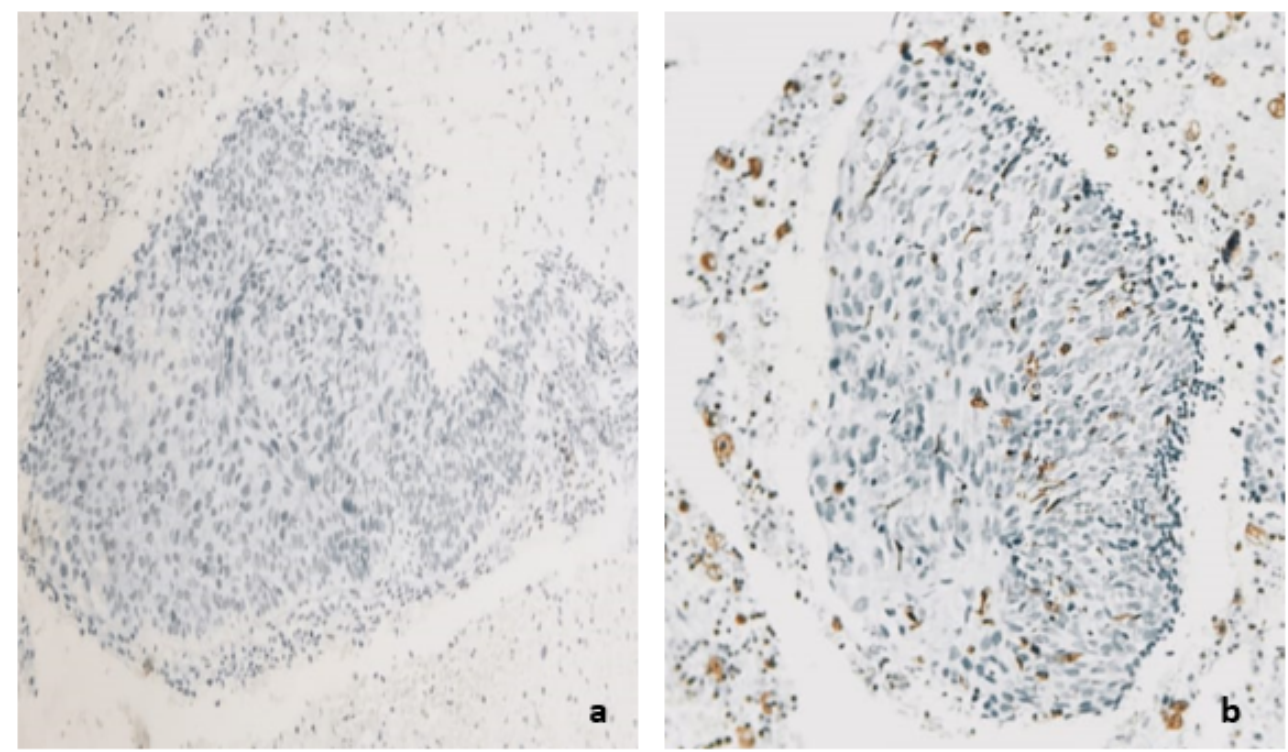

Figura 3. Inmunohistoquímica Receptores de estrógenos (RE) y Vimentina. Se observan cortes de tejido endometrial con ausencia de tinción para receptores de estrógeno en las células tumorales (a). Ausencia de tinción para vimentina en las células tumorales (b). Inmunohistoquímica, receptores de estrógeno, vimentina, $40 X$.

\section{DISCUSIÓN}

La EXG es un hallazgo histológico raro, que se caracteriza por la presencia de histiocitos espumosos cargados de lípidos asociados a un infiltrado inflamatorio mixto que borra la arquitectura normal del endometrio. Según la literatura consultada, sólo hay 24 casos de esta entidad reportados en la literatura (3). La EXG se presenta comúnmente en pacientes entre los 45 y 88 años de edad, con predominio en las pacientes postmenopáusicas (4). Su debut clínico se caracteriza por dolor abdominal, hemorragia uterina anormal, flujo vaginal e incluso masa pélvica palpable (5). Si bien se conocen algunos factores de riesgo, la fisiopatología de esta entidad no es clara. Se plantea que hay una interacción compleja entre múltiples factores, entre los que se encuentran la obstrucción del cérvix, la inflamación, y la presencia de lípidos, lo que lleva a la generación de radicales libres y la peroxidación lipídica (6).

En nuestra paciente, una vez realizado el diagnóstico de EXG asociado a un carcinoma mal diferenciado, se procedió a ejecutar el abordaje inmunohistoquímico con el fin de confirmar y/o descartar carcinoma endometrial, adenocarcinoma de endocérvix o carcinoma escamocelular. El carcinoma de endometrio es positivo para receptores de estrógeno, receptores de progesterona y vimentina (7). El adenocarcinoma de endocérvix es positivo para p16 y negativo para $\mathrm{p} 63$, el carcinoma escamocelular es positivo para citoqueratinas, p63 y p16 (8). Es nuestro caso, los receptores de estrógenos y vimentina fueron negativos y la tinción con citoqueratinas, p63 y p16 fueron positivas, confirmando el origen escamocelular del carcinoma. Según la literatura consultada, hay nueve casos de EXG que se asociaron con procesos neoplásicos malignos. De estos, siete casos se asociaron con carcinoma de endometrio, un caso con carcinoma mixto de endometrio y uno con carcinoma escamocelular de cérvix (6); los casos restantes en la literatura no se asociaron con malignidad (3). Es imposible diferenciar clínica e imagenológicamente la inflamación xantogranulomatosa de endometrio de una neoplasia maligna, dado que la EXG puede cursar con linfadenopatías pélvicas e inguinales reactivas, por lo cual, su correlación clínico patológica es definitoria (9). Se reconoce que la diabetes mellitus es un factor de riesgo para que se 
desarrollen procesos inflamatorios xantogranulomatosos en diferentes órganos (10).

Los pacientes diabéticos presentan disfunción leucocitaria, alteración en la quimiotáxis, la adherencia y fagocitosis. Según lo anterior, y correlacionado con nuestra paciente, el hecho de presentar un proceso secundario como el carcinoma escamocelular, que por sí sólo puede ocasionar estenosis cervical asociado a la diabetes de base, podría explicar el proceso inflamatorio xantogranulomatoso del endometrio.

Cuando el médico patólogo se enfrente a un caso de endometritis xantogranulomatosa, debe descartar un proceso neoplásico concomitante hasta que se demuestre lo contario, principalmente un carcinoma de endometrio. En cuanto a los diagnósticos diferenciales, la EXG no debe ser confundida con una malacoplaquia, histiocitosis de células de Langerhans, o más frecuente con un cambio decidual del endometrio. La malacoplaquia se caracteriza por el hallazgo patognomónico de los cuerpos de Michaelis-Gutmann (11), en la histiocitosis de células de Langerhans se observará tinción para CD1a y CD207 (Langerina), mientras que en la EXG los histiocitos espumosos serán positivos para CD68 (12). En el cambio decidual secundario a una fase secretora tardía, o a un estímulo progestacional externo, las células deciduales serán de tamaño intermedio con núcleos redondos, excéntricos, de citoplasmas amplios y membranas citoplasmáticas bien definidas asociado a la morfología clásica de las glándulas secretoras, en contraste con el borramiento arquitectural que ocasiona la EGX y la presencia de los histiocitos espumosos (13).

\section{CONCLUSIONES}

La EXG es una entidad rara en el endometrio, la cual debe obligar al médico patólogo a descartar una entidad neoplásica maligna concomitante, debido a la asociación con carcinomas endometriales y cervicales, ayudándose siempre de la morfología y utilizando para su confirmación estudios complementarios de inmunohistoquímica.
CONFLICTO DE INTERÉS: Ningún conflicto de interepes que declarar.

\section{REFERENCIAS}

1. Goodman, M., Curry, T. and Russell, T., 1979. Xanthogranulomatous Pyelonephritis (XGP). Medicine, 58(2), pp.171-181.

2. Guzman-Valdivia G. Xanthogranulomatous cholecystitis: 15 years'experience. World J Surg 2004; 28: 254-7.

3. Du XZ, Lu M, Safneck J, Baker P, Dean E, Mottola J. Xanthogranulomatous endometritis mimicking endometrial carcinoma: A case report and review of literature. Radiol Case Rep. 2019 Jan;14(1) 121-125.

4. Noak F, Briese J, Stellmacher F, Hornung D, Horny H. Lethal outcome in xanthogranulomatous endometritis. APMIS 2006;114(5):386-8 May.

5. Gami N, Mundhra R, Guleria K, Arora VK, Garg S. Recurrent pyometra and xanthogranulomatous salpingitis: a rare pathologic association in a postmenopausal lady. J Midlife Health 2014;5(3):156-8.

6. Russack V, Lammers RJ. Xanthogranulomatous endometritis. Report of six cases and a proposed mechanism of development. Arch Pathol Lab Med 1990;114(9):929-32 Sep.

7. Murali R, Davidson B, Fadare O, et al. Highgrade Endometrial Carcinomas: Morphologic and Immunohistochemical Features, Diagnostic Challenges and Recommendations. Int J Gynecol Pathol. 2019;38 Suppl 1(Iss 1 Suppl 1): S40-S63.

8. Rutgers JK, Roma AA, Park KJ, et al. Pattern classification of endocervical adenocarcinoma: reproducibility and review of criteria. Mod Pathol. 2016;29(9):1083-1094. 
9. Badhe PB. Xanthogranulomatous endometritis. Indian J Pathol Microbiol 1996;39(4):321-3 Oct.

10. Kitagawa S, Nakagawa M, Yamada T, Mori Y, Simizu H, Rin S, et al. Clinico-pathological study of xanthogranulomatous cholecystitis. Nihon Geka Gakkai Zasshi 1990;91(8):1001-10 Aug.

11. Abolhasani M, Jafari AM, Asgari M, Salimi $\mathrm{H}$. Renal malakoplakia presenting as a renal mass in a 55-year-old man: a case report. J Med. 2012; 6: 379 .

12. Wader JV, Jain A, Kumbhar SS, Vhawal V. Histiocytic endometritis. Am J Case Rep 2013; 14: 329-32 Aug 26.

13. Zhang XS, Dong HY, Zhang LL, Desouki MM, Zhao C. Xanthogranulomatous inflammation of the female genital tract: report of three cases. J Cancer 2012; 3: 100-6. 